\title{
FLAMBAGEM VERTICAL EM TUBULAÇÕES DE DISTRIBUIÇÃO DE GÁS NATURAL
}

\author{
J. C. ALMEIDA \\ Companhia Paranaense de Gás - COMPAGAS; PGMec - Programa de Pós-Graduação em Engenharia Mecânica \\ da Universidade Federal do Paraná \\ j.cezar@compagas.com.br; j.cezar@ufpr.br
}

Submetido 10/10/2016 - Aceito 12/12/2016

DOI: $10.15628 /$ holos.2017.5187

\section{RESUMO}

Tubulações de aço são normalmente utilizadas em sistemas de transmissão e também em redes de distribuição de gás natural. Estas estruturas, quando em operação, são submetidas a cargas externas, que quando atingem valores críticos, podem ocasionar falhas, até mesmo catastróficas. Os acidentes, nestas circunstâncias, podem resultar em prejuízos muito elevados, danos ambientais e até mesmo problemas de imagem para a empresa envolvida. No caso específico das redes de transmissão e distribuição de gás natural, o projeto e construção seguem rígidos padrões, entre as quais podem se destacar a norma NBR-12712. A combinação de altas temperaturas e pressões internas aos quais os dutos podem ser submetidos causa expansões, as quais são restringidas pela ação solo, gerando assim forças axiais de compressão. Neste contexto, o tubo pode entrar em colapso através do fenômeno conhecido como flambagem vertical ou flambagem horizontal. No entanto, como essas tubulações são normalmente enterradas, elimina-se a preocupação com a flambagem horizontal permanecendo, entretanto, uma maior preocupação com a flambagem vertical assim existente. O objetivo do presente trabalho é determinar, de forma conceitual, as temperaturas limites que uma malha de distribuição de gás natural pode ser submetida, sem apresentar problemas de flambagem vertical. Este estudo é realizado com os dados operacionais da rede de distribuição de gás natural da COMPAGAS - empresa de distribuição de gás natural ou responsável no estado do Paraná. Para tanto se utilizou equações matemáticas simplificadas para o estudo da flambagem em dutos verticais aquecidos, seguindo o modelo proposto $\mathrm{e}$ adaptado por Hobbs.

PALAVRAS-CHAVE: Hobbs, flambagem vertical, rede de distribuição de gás natural, dutos enterrados.

\begin{abstract}
Steel pipes are commonly used in transmission systems and also in natural gas distribution networks. These structures, when in operation, are subjected to external loads, which when they reach critical values, may cause failures, even catastrophic. Accidents in these circumstances may result in very high losses, environmental damage and even image problems for the company involved. In the specific case of networks of transmission and distribution of natural gas, the design and construction follow strict standards, among which we can highlight the NBR 12712 standard. The combination of high temperatures and internal pressures to which the products may be subjected to expansions, which are restricted by soil action, thus creating axial compression forces. In this context, the
\end{abstract}

tube may collapse via buckling phenomenon known as vertical or horizontal buckling. However, as these pipes are usually buried, it eliminates the concern with the horizontal buckling remaining, however, a greater concern with the existing vertical well buckling. The objective of this study is to determine, conceptually, the temperature limits that a natural gas distribution mesh can be submitted without providing vertical buckling problems. This study is conducted with the operational data of the network of natural gas distribution COMPAGAS - distribution company of natural gas or responsible in Parana state. For this simplified mathematical equations are used for the study of buckling in heated vertical ducts, following the model and adapted by Hobbs.

KEYWORDS: Hobbs, vertical buckling, natural gas distribution network, buried pipes. 


\section{INTRODUÇÃO}

Tubulações ou dutos vêm sendo amplamente empregados mundialmente para o transporte de petróleo, gás e seus derivados. Tais dispositivos operam, quase que na sua totalidade, enterrados em profundidades que variam de acordo com as condições de segurança e com as normas correspondentes para cada caso específico, motivo pelo qual os mesmos se encontram em contato direto com o terreno local e consequentemente ficam susceptíveis às condições impostas pelo mesmo.

Essas estruturas, quando em operação, são submetidas a cargas externas que quando atingem valores críticos podem ocasionar diversos tipos de falhas, às vezes catastróficas. Segundo Almeida (2013), acidentes, nessas circunstâncias, resultam em elevadas perdas econômicas, danos ambientais e até mesmo problemas de imagem para com a instituição envolvida, gerando assim a necessidade de que os projetos sejam cada vez mais seguros e eficazes seguindo padrões e normas internacionais.

Para o caso específico de redes de transmissão e distribuição de gás natural, o projeto e construção seguem a norma brasileira NBR-12712, a qual corresponde em diversos trechos, a uma cópia da norma americana ANSI B.31.8.

Durante o período de operação, os dutos podem vir a ser submetidos a altas temperaturas e pressões internas. Tais condições causam a expansão do duto, a qual é combatida pela restrição imposta pelo solo ao livre deslocamento axial do mesmo, gerando forças axiais de compressão (Dvorkin \& Toscano, 2011). Caso estas forças compressivas atinjam valores críticos, o duto poderá sofrer colapso devido à flambagem, seja no plano vertical ou no plano horizontal, conforme indicado nas Figuras 1 e 2, respectivamente.

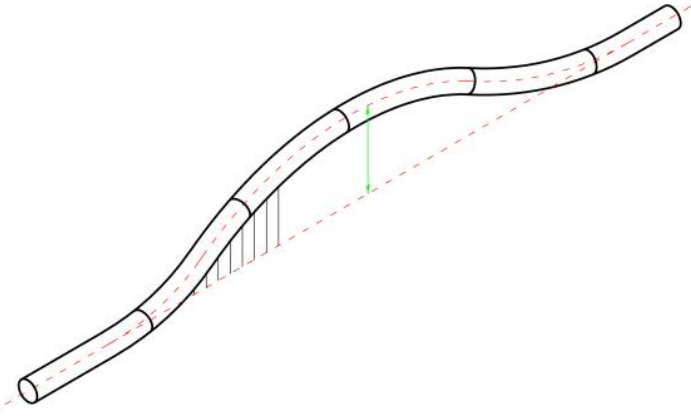

Figura 1: Flambagem vertical de um duto.

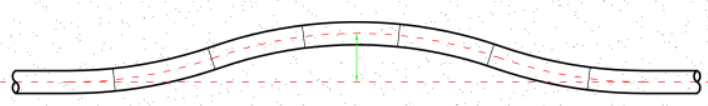

Figura 2: Flambagem lateral de um duto.

Segundo Hobbs (1984), a ocorrência deste fenômeno, no plano horizontal ou vertical, depende da contenção fornecida pelo solo e pelo peso efetivo do duto. Para dutos superficiais, a flambagem lateral ocorre primeiramente devido ao efeito do peso do duto. Por outro lado, para dutos enterrados em profundidades maiores, a contenção do solo praticamente elimina a flambagem lateral e o problema passa ser exclusivo em termos da flambagem vertical.

Especificamente para o caso de dutos de distribuição de gás natural em aço carbono, os mesmos normalmente operam em profundidades médias de 1,20 $\mathrm{m}$ a partir da geratriz superior do duto, justificando assim a proposta de estudo para o fenômeno desta perda de estabilidade do duto. 


\section{CONCEITUAÇÃO TEÓRICA}

Diferentemente da flambagem convencional, a qual ocorre com pilares e peças esbeltas, a flambagem em dutos corresponde a um fenômeno bem mais complexo, dado que em pilares o comprimento efetivo de flambagem é um dado inicial do problema, dependendo ainda do tipo e das posições dos apoios correspondentes. Por outro lado, para dutos o comprimento efetivo de flambagem é uma variável a priori desconhecida, sendo determinada apenas ao final do problema (Grangeiro, 2009b). Isso ocorre porque os deslocamentos axiais e transversais do duto são restringidos pelo solo, impedindo a flambagem global da estrutura e transformando a flambagem de dutos num fenômeno localizado em apenas um pequeno trecho do duto (Grangeiro, 2009).

O modelo de Hobbs para dutos se aplica para o estudo da flambagem vertical de dutos, na qual o mesmo é modelado como uma viga longa sobre uma base rígida em que a interação soloestrutura é representada pela ação do atrito na direção axial e pelo peso da cobertura do solo no sentido vertical. Através desse modelo e de expressões simples, torna-se assim possível obter a temperatura segura de operação.

Considera-se assim o duto como uma viga inicialmente reta submetida a uma carga vertical uniformemente distribuída (peso próprio $(W)+$ cobertura do solo) e às forças axiais de compressão devido à restrição do solo à expansão térmica do duto, sendo que após a flambagem o mesmo assume a configuração ilustrada na Figura 3. Observar que esse modo de flambagem é simétrico em relação à origem.

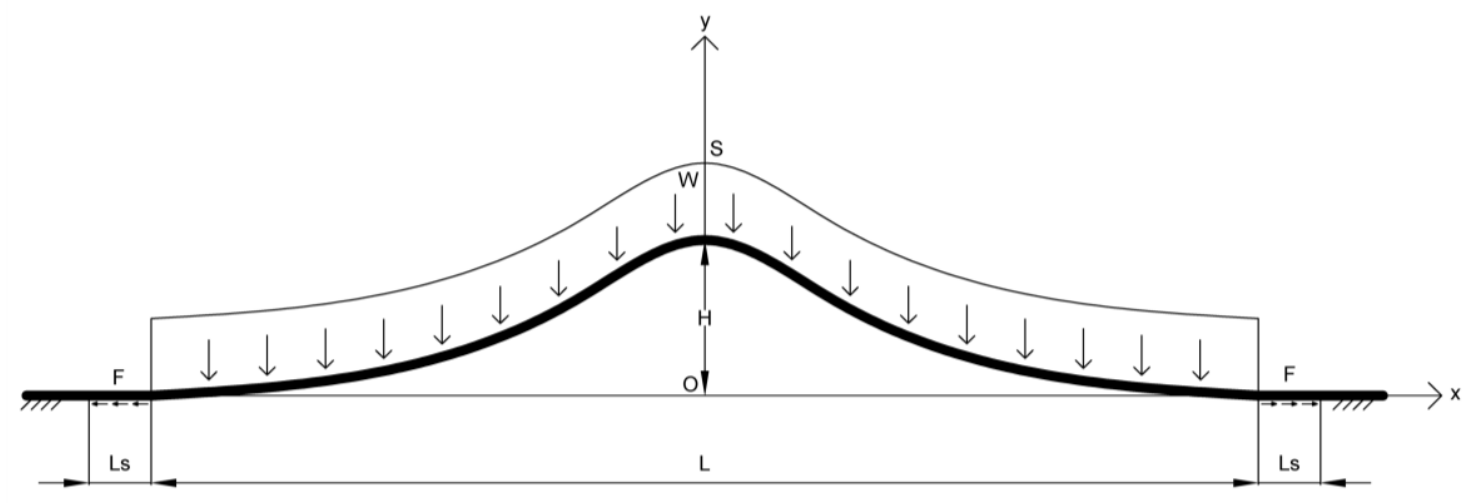

Figura 3: Modelo de base rígida para flambagem vertical (adaptado de Neto, 2009).

A equação diferencial que rege o comportamento do modelo descrito é obtida através da utilização da teoria clássica de flexão de vigas-colunas (Chajes, 1974) com a consideração da atuação simultânea de uma carga distribuída e uma força axial compressiva. Matematicamente, tem-se:

$\frac{d^{4} y}{d x^{4}}+\lambda^{2} \frac{d^{2} y}{d x^{2}}+\frac{W}{E I}=0$ 
sendo o índice de esbeltez $(\lambda)$ dado por:

$\lambda=\sqrt{\frac{P}{E I}}$

Aplicando-se as condições de contorno, conforme Figura 3, obtém-se a solução da equação viga-coluna na forma:

$$
y=\frac{W E I}{P^{2}}\left[1+\frac{\lambda^{2} L^{2}}{8}-\frac{\lambda^{2} x^{2}}{2}-\frac{\cos \lambda x}{\cos (\lambda L / 2)}\right]
$$

A referida equação apresenta duas variáveis desconhecidas - $\lambda$ e L. Aplicando-se, porém, a condição de contorno correspondente à rotação nos apoios $\left(y^{\prime}=0\right)$, pode-se chegar especificamente a:

$\tan \left(\frac{\lambda L}{2}\right)=\frac{\lambda L}{2} \Rightarrow \lambda L=8,9868$

cuja solução, para a condição crítica da carga $\mathrm{P}$, corresponde a:

$P=80,76 \frac{E I}{L^{2}}$

Caso o comprimento $L$ fosse inicialmente conhecido o problema estaria finalizado. Entretanto e conforme descrito anteriormente, a flambagem de dutos longos correspondente a um fenômeno localizado, envolvendo apenas um pequeno trecho do comprimento total, fazendo assim com que o comprimento da flambagem seja uma variável do problema, cuja determinação exija equações adicionais de equilíbrio e compatibilidade.

\subsection{Força atrito entre duto e solo}

Para a condição de um coeficiente de atrito $(\mu)$, supostamente constante entre o solo e o duto, pode-se considerar a existência de uma força de atrito $F$ atuante ao longo de um comprimento $L s$ de cada lado à região adjacente ao modo de flambagem, conforme representado no modelo da Figura 3. Conceitualmente, porém, três casos específicos de análise podem ser abordados nesse contexto:

a) a não existência de deslizamento na região longitudinal de contato (touchdown points), acarretando numa mudança brusca entre a carga axial $P$ na região de flambagem e a carga axial $N$ fora desta região;

b) a existência de atrito na região de contato;

c) a não consideração da força de atrito nos pontos concentrados. 
Para os três casos descritos torna-se possível determinar o comprimento de flambagem correspondente. Na prática, porém, pode-se considerar a avaliação dos comprimentos de flambagem a partir dos casos extremos apresentados - (a) e (c) (Grangeiro \& Júnior, 2009a), dado que o caso intermediário (b) traria valores entre os limites extremos definidos.

\subsection{Carga axial crítica}

A carga axial crítica corresponde à menor carga necessária para que se mantenha o duto na posição flambada. A mesma é obtida a partir do alongamento do duto ocasionado pela variação de temperatura existente. Tal variação pode ser equacionada pela diferença entre o comprimento do duto curvo, pós-flambagem $(S)$ e a distância entre os pontos que a tubulação se eleva $(L)$. Matematicamente, para o caso $(c)$ :

$$
S-L=\int_{-L / 2}^{L / 2} \frac{1}{2}\left(y^{\prime}\right)^{2} d x=75,60 \frac{W^{2}(E I)^{1,5}}{P^{3,5}}
$$

resultando, mediante uma boa quantidade de manipulações matemáticas, em:

$$
N=80,76 \frac{E I}{L^{2}}+\frac{W L^{3,5}}{E I} \sqrt{15,97 \cdot 10^{-6} F E A}
$$

Nessas circunstâncias, o comprimento de flambagem decorre da condição $(d N / d L=0)$ imposta à equação (7), chegando-se finalmente a:

$$
L_{C}=\sqrt[11]{3,31.10^{7} \frac{(E I)^{4}}{W^{2} E A}}
$$

De forma similar, torna-se também possível obter a carga crítica de flambagem e o correspondente comprimento de flambagem para o caso (a), ou mais especificamente:

$$
\begin{aligned}
& N=80,76 \frac{E I}{L^{2}}+15,97 \cdot 10^{-6} \frac{E A W^{2} L^{6}}{(E I)^{2}} \\
& L_{A}=\sqrt[8]{16,825 \frac{(E I)^{3}}{W^{2} E A}}
\end{aligned}
$$

\subsection{Efeito da pressão interna e da temperatura}

As forças axiais atuantes no duto podem ser de duas fontes distintas, sendo a primeira referente à dilatação térmica e a segunda ao efeito da pressão interna. Para o caso da temperatura, vale a relação numérica: 


$$
N=E A \alpha \Delta T \Rightarrow \Delta T_{S E G}=\frac{N_{C R I T}}{E A \alpha}
$$

de forma que ao se utilizar a carga crítica $N_{C R I T}$, pode-se obter a variação de temperatura correspondente, a qual define a faixa na qual o duto poderá operar de maneira segura com relação à flambagem térmica $\left(\Delta \mathrm{T}_{\mathrm{SEG}}\right)$.

Para o caso da pressão interna, aplica-se a Lei de Hooke generalizada envolvendo a deformação longitudinal axial para um tubo de parede fina, ou mais especificamente:

$$
\varepsilon_{a}=\frac{1}{E}\left(\sigma_{a}-v \sigma_{\theta}\right)=\frac{1}{E}\left(\frac{p r}{2 t}-v \frac{p r}{t}\right)
$$

Tal deformação ao ser impedida pelo solo gera um esforço compressivo dado por:

$$
N_{p}=E A \varepsilon_{a}=\frac{A p r}{t}\left(\frac{1}{2}-v\right)
$$

A combinação das equações (12) e (13) possibilita, por fim, descrever uma equação que permita determinar o aumento equivalente de temperatura devido à pressão interna:

$$
\Delta T_{p}=\frac{p r}{t E \alpha}\left(\frac{1}{2}-v\right)
$$

Tal valor deve, ainda, ser deduzido da variação da temperatura obtida a partir da equação (13), de tal sorte que a segurança do duto seja realizada mediante a comparação do aumento de temperatura efetiva $\left(\Delta \mathrm{T}_{\mathrm{SEG}}{ }^{\text {efet }}\right)$ com a temperatura considerada segura em relação à flambagem vertical, ou mais especificamente:

$$
\Delta T_{S E G}{ }^{\text {efet }}=\Delta T_{S E G}-\Delta T_{p}
$$

\section{A REDE DA COMPAGAS - PARÂMETROS DE CÁLCULO}

A COMPAGAS corresponde à empresa estatal responsável pela distribuição de gás natural no Estado do Paraná, Brasil. A mesma tem cerca de 18 anos de operação e dispõe, atualmente, de uma extensão aproximada de $700 \mathrm{~km}$, contemplando tubulações de aço carbono e PEAD (polietileno de alta densidade) ao longo de nove municípios distintos. Para o presente estudo considerou-se apenas as tubulações de aço carbono, face às condições e premissas operacionais envolvidas.

Para essa condição, a COMPAGAS dispõe de tubulações com diâmetros nominais que variam de 4" a 10", operando com pressões nominais de 4, 7, 17 e 35 bar. A Tabela 1 apresenta as características geométricas e as faixas de pressão operacionais das redes de distribuição. Para o aço carbono (API 5L Gr.B), considerou-se: $\mathrm{E}=207 \mathrm{GPa}, v=0,29$ e $\alpha=12 \cdot 10^{-6}\left({ }^{\circ} \mathrm{C}\right)^{-1}$. 
Tabela 1: Parâmetros geométricos e faixas de pressão - tubulações utilizadas pela COMPAGAS.

\begin{tabular}{c|c|c|c|c}
\hline $\begin{array}{c}\text { Diâmetro } \\
\text { nominal (in) }\end{array}$ & $\begin{array}{c}\text { Espessura de } \\
\text { parede }(\mathrm{mm})\end{array}$ & $\begin{array}{c}\text { Diâmetro } \\
\text { interno }(\mathrm{mm})\end{array}$ & $\begin{array}{c}\text { Pressões de } \\
\text { trabalho (bar) }\end{array}$ & $\begin{array}{c}\text { Peso próprio } \\
(\mathrm{N} / \mathrm{m})\end{array}$ \\
\hline $4 "$ & 6,02 & 102,26 & 4,7 ou 17 & 157,70 \\
$6 "$ & 4,80 & 158,70 & $4,7,17,35$ & 189,87 \\
$8^{\prime \prime}$ & 4,80 & 209,50 & $7,17,35$ & 248,86 \\
$10^{\prime \prime}$ & 6,40 & 260,30 & $7,17,35$ & 412,94 \\
\hline
\end{tabular}

\section{RESULTADOS E DISCUSSÕES - CARGA CRÍTICA E COMPRIMENTO DE FLAMBAGEM}

A partir da formulação descrita definiu-se as temperaturas consideradas supostamente seguras para a operação de cada duto em função do seu diâmetro e da faixa de pressão ao qual o mesmo é submetido. Esses resultados foram definidos na Tabela 3.

Tabela 2: Cargas críticas e comprimentos de flambagem - Casos (a) e (c).

\begin{tabular}{c|c|c|c|c}
\hline $\begin{array}{c}\text { Diâmetro } \\
\text { nominal (in) }\end{array}$ & $\mathrm{L}_{\mathrm{A}}(\mathrm{m})$ & $\mathrm{N}_{\text {crit }}(\mathrm{kN})$ & $\mathrm{L}_{\mathrm{C}}(\mathrm{m})$ & $\mathrm{N}_{\text {crit }}(\mathrm{kN})$ \\
\hline $4^{\prime \prime}$ & 4,99 & 2018,81 & 40,44 & 91,02 \\
6" & 6,80 & 2985,05 & 55,46 & 141,10 \\
8" $^{\prime \prime}$ & 8,32 & 4478,89 & 69,19 & 224,22 \\
$10^{\prime \prime}$ & 9,81 & 8291,21 & 84,95 & 460,67 \\
\hline
\end{tabular}

Tabela 3: Faixas de temperaturas para a faixa de diâmetros da COMPAGAS - Casos (a) e (c).

\begin{tabular}{c|c|c|c}
\hline $\begin{array}{c}\text { Diâmetro } \\
\text { nominal (in) }\end{array}$ & $\begin{array}{c}\text { Pressão de } \\
\text { trabalho (bar) }\end{array}$ & $\begin{array}{c}\Delta \mathrm{T}_{\mathrm{SEG}}{ }^{\text {efet }}\left({ }^{\circ} \mathrm{C}\right) \\
\text { Caso (a) }\end{array}$ & $\begin{array}{c}\Delta \mathrm{T}_{\mathrm{SEG}}{ }^{\text {efet }}\left({ }^{\circ} \mathrm{C}\right) \\
\text { Caso (c) }\end{array}$ \\
\hline $4 "$ & 4,0 & 396,58 & 17,606 \\
$4 "$ & 7,0 & 396,37 & 17,390 \\
$4 "$ & 17,0 & 395,65 & 16,672 \\
$6 "$ & 4,0 & 486,85 & 22,481 \\
$6 "$ & 7,0 & 486,43 & 22,062 \\
$6 "$ & 17,0 & 485,03 & 20,664 \\
$6 "$ & 35,0 & 482,52 & 18,148 \\
$8 \prime$ & 7,0 & 556,67 & 26,641 \\
$8 \prime$ & 17,0 & 554,83 & 24,796 \\
$8^{\prime \prime}$ & 35,0 & 551,51 & 21,475 \\
$10^{\prime \prime}$ & 7,0 & 621,26 & 33,381 \\
$10^{\prime \prime}$ & 17,0 & 619,54 & 31,662 \\
$10^{\prime \prime}$ & 35,0 & 616,45 & 28,567 \\
\hline
\end{tabular}

Pelos valores obtidos e como as faixas de temperaturas para o caso (a) superam uma variação de quase 400 ㄷ $\mathrm{C}$, torna-se evidente que o caso (c), ou mais especificamente, à condição na qual não se considera a força de atrito localizada na formulação matemática, corresponde à situação crítica em termos das faixas de temperatura a serem consideradas para fins de 
avaliações e comparações finais. A Figura 4 ilustra um gráfico representativo da variação da temperatura em função da faixa de diâmetros nominais disponíveis e das faixas de pressão operacionais correspondentes. Do mesmo observa-se que as situações operacionais críticas decorrem do uso de diâmetros menores, bem como, da operação em pressões mais elevadas, conforme esperado.

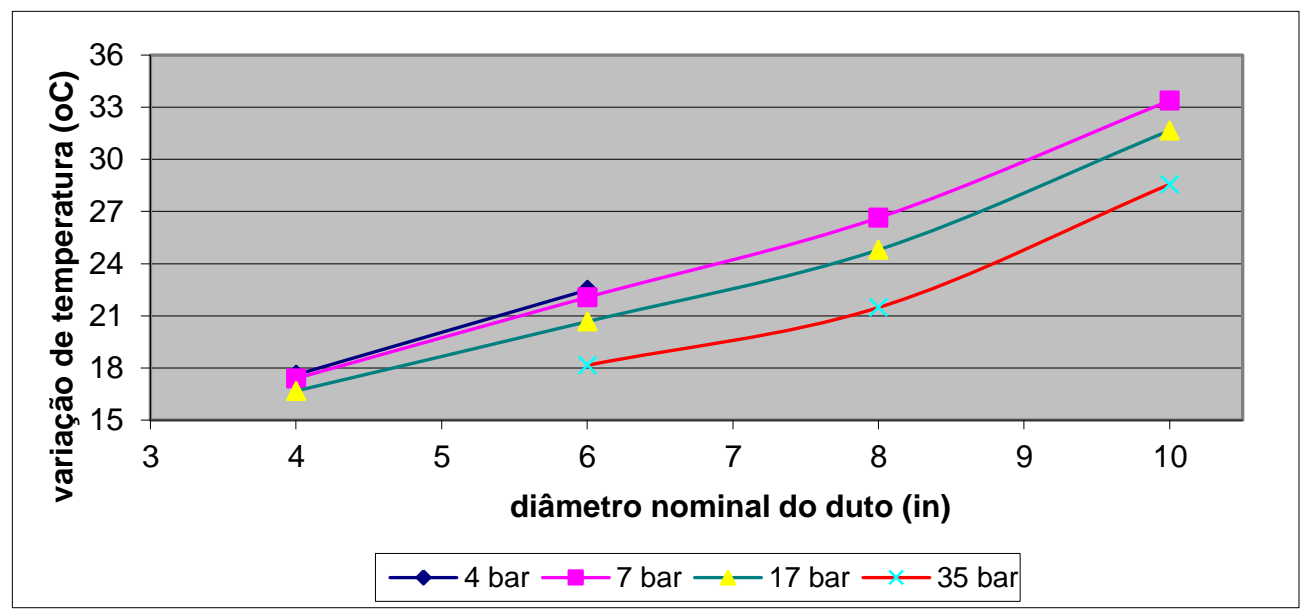

Figura 4: Variação da temperatura com as pressões de trabalho e diâmetros nominais da tubulação - Caso (c).

Pode-se também concluir que, num contexto operacional geral, no qual as redes de distribuição de gás natural operam com pressões máximas na faixa dos 35 bar, como também, não apresentam diâmetros nominais inferiores a 4" (exceto em situações de pequenos ramais de serviço), o problema da flambagem vertical é fator pouco significativo no projeto desse tipo de estrutura. Tal informação se confirma a partir dos valores obtidos para as faixas de temperatura, supostamente mais críticas, ou ainda variações de temperatura próximos dos $17^{\circ} \mathrm{C}$. Na prática, ainda, pode-se supor que a variação de temperatura ao longo do fluido em tubulações enterradas a profundidades próximas de 1,0 m, acompanhe a variação da temperatura ambiente, justificando ainda mais a margem de segurança envolvida nesse contexto.

\section{CONCLUSÕES}

Os resultados matemáticos obtidos (conforme Tabela 3) para a condição, supostamente, mais crítica em termos do fenômeno da flambagem vertical em dutos convencionais de distribuição de gás natural de aço carbono, demonstram a pouca probabilidade desse problema vir a comprometer as condições operacionais correspondentes, dado que: para o caso (a) as variações de temperatura superam quase os $400 \circ \mathrm{C}$ e para o caso (c) a condição operacional mais crítica corresponde à uma variação de temperatura de $16,672^{\circ}$ C quando se considera dutos de 4" de diâmetro operando em pressões de 17 bar.

Considerando ainda a quase totalidade das empresas de distribuição de gás natural no Brasil, pode-se afirmar que as mesmas trabalham com pressões operacionais e faixas de diâmetros equivalentes aos valores utilizados no presente estudo, caracterizando com isso a pequena influência do problema da flambagem vertical em situações operacionais desta natureza.

Por fim, evidente afirmar que para situações pontuais contemplando tubulações de aço carbono com diâmetros de menor dimensão e que venham a operar com pressões mais elevadas (de 17 bar para mais) sugere-se uma avaliação mais criteriosa em relação ao referido fenômeno. 


\section{NOMENCLATURA E ABREVIATURAS}

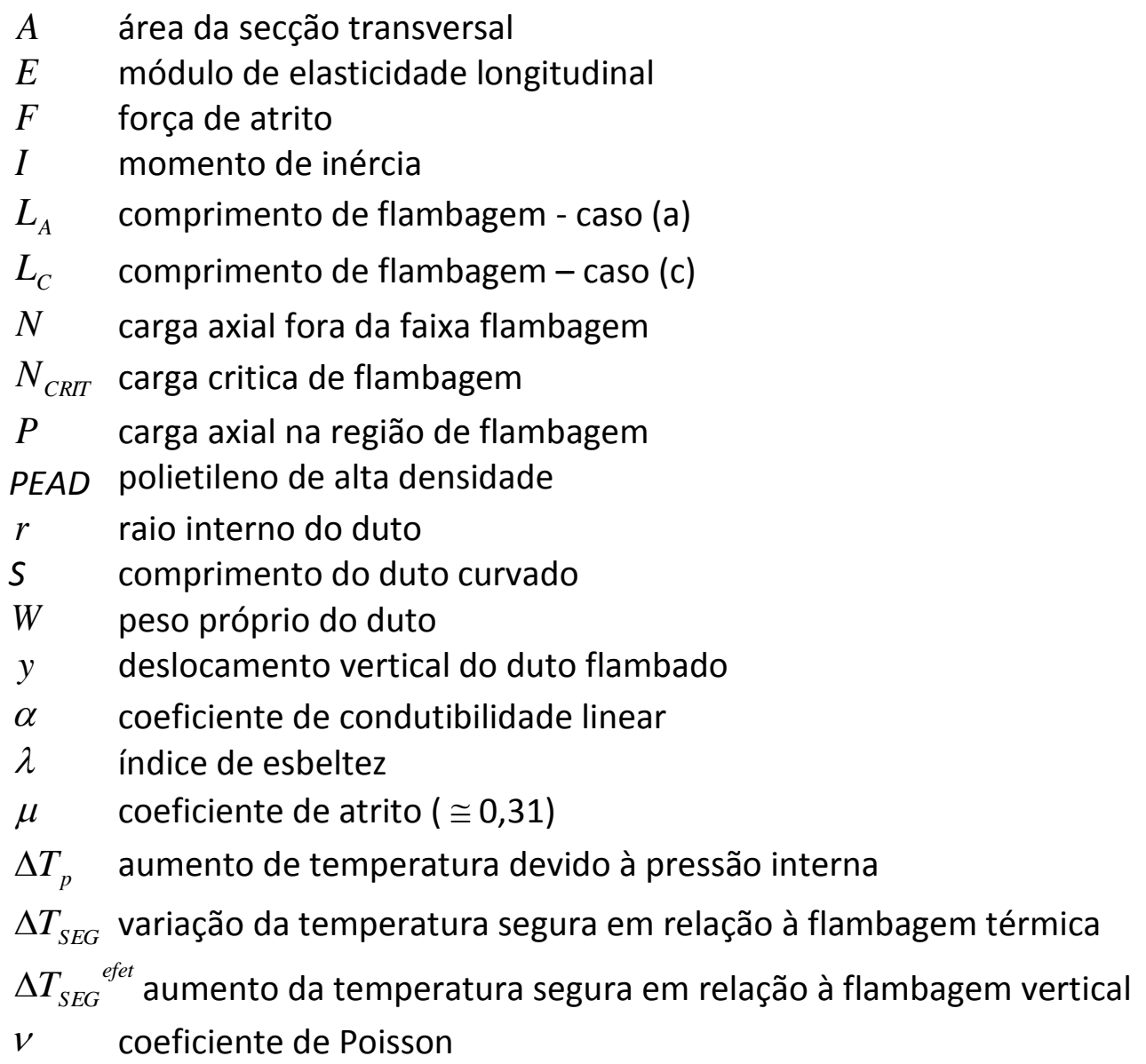

\section{REFERÊNCIAS}

ABNT, NBR 12712. (2002). Projeto de Sistemas de Transmissão e Distribuição de Gás Combustível. Associação Brasileira de Normas Técnicas.

Almeida, J. C. (2014). Estudo numérico e experimental de escoamentos transitórios em redes de distribuição de gás natural. Tese de Doutorado, Pontifícia Universidade Católica do Paraná, Curitiba, PR, Brasil.

Chajes, A. (1974). Principles of structural stability theory. Prentice-Hall, Englewoord Cliffs.

Dvorkin, E.N., \& Toscano, R.G. (2011). Effects of internal/external pressure on the global buckling of pipelines. Elsevier Science Ltd. Computational Fluid and Solid Mechanics.

Grangeiro, A.E.B. (2009b). Um estudo da flambagem vertical de dutos através de modelos analíticos e computacionais. Trabalho de Monografia, Universidade Federal do Ceará, Fortaleza, $\mathrm{CE}$, Brasil.

Grangeiro, A.E.B.; \& Júnior, E.P. (2009a). Flambagem vertical de dutos submetidos a aumento de temperatura. 5ㅇ Congresso Brasileiro de Petróleo e Gás. Universidade Federal do Ceará , Fortaleza, CE, Brasil.

Hobbs, R.E. (1984). In service buckling of heated pipelines. Journal of transportation engineering, ASCE, vol. 110, n.2, pp. 175-189.

Neto, L.B. (2009). Análise numérica de dutos sujeitos à flambagem. Dissertação de mestrado, Universidade Federal do Paraná, Curitiba, PR, Brasil. 